\title{
Design of a High Voltage Multiplier with Series- Connected Bipolar Topology
}

\author{
Kei Eguchi, Kanji Abe, Hiroki Fujisawa, Ichirou Oota
}

\begin{abstract}
Recently, non-thermal food processing utilizing an underwater shockwave has been receiving much attention to provide nutritious and fresh foods at low cost. To generate the underwater shockwave, we propose a series-connected high voltage multiplier with bipolar structure. Unlike conventional Cockcroft-Walton voltage multipliers (CWVMs), the proposed multiplier amplifies the input voltage twice by connecting positive/negative multiplier blocks in series. Furthermore, these multiplier blocks are driven by high speed rectangular pulses. Owing to the series-connected bipolar topology, the proposed multiplier can achieve not only high conversion ratio but also high response speed. The operation principle and characteristic evaluation are described concerning the proposed multiplier. Simulation program with integrated circuit emphasis (SPICE) simulations demonstrate the feasibility and effectiveness of the proposed multiplier.
\end{abstract}

Keywords - bipolar topology, Cockcroft-Walton multipliers, high speed multipliers, non-thermal food processing, seriesconnected multipliers.

\section{Introduction}

In recent years, non-thermal food processing utilizing an underwater shockwave [1]-[4] is one of the most promising techniques to provide nutritious and fresh foods at low cost. In the non-thermal food processing, the cell wall and organization of foods are destroyed by the high-speed destruction phenomenon that is called spalling destruction [1][4]. To generate the underwater shockwave, the high voltage multiplier, such as the Cockcroft-Walton voltage multiplier (CWVM) [5], is commonly used to cause the spalling destruction. The benefits of the CWVM are as follows: 1. by increasing the number of stages, a high stepped-up voltage can be generated easily; and 2 . unlike a high voltage transformer with high turn ratio, the CWVM can achieve light weight and small size, because the CWVM requires no magnetic components.

Kei Eguchi, Kanji Abe, Hiroki Fujisawa

Fukuoka Institute of Technology

Japan

Ichirou Oota

National Institute of Technology, Kumamoto College Japan
In past studies, several attempts have been made to develop the efficient CWVM. For example, Wang et al. exhibited a cascade CWVM and its model of parasitic capacitances [6] in 2013. However, it requires a transformer with center-tapped secondary to perform its push-pull kind of operation. Iqbal and Besar proposed a bipolar CWVM [7] in 2007. Unlike a voltage multiplier using center-tapped transformers, the bipolar CWVM reported in [7] requires only one ac power source. Following this study, a hybrid symmetrical CWVM [8] was suggested by Iqbal in 2014. Owing to the hybrid symmetrical topology, the hybrid symmetrical CWVM reported in [8] can improve the voltage drop and transient response from the conventional converter reported in [7]. However, the response speed of these conventional CWVMs is still slow, because the diode switch is driven by a commercial ac power source. To improve the response speed of CWVMs, we proposed a parallel-connected high-speed voltage multiplier [9] in 2014. Using high/low side drivers, the diode switch of the CWVM reported in [9] is driven by high speed rectangular pulses. Therefore, the CWVM reported in [9] can achieve not only high step-up conversion ratio but also high response speed. However, the number of stages is still large.

In this paper, a novel CWVM with series-connected bipolar topology is proposed for non-thermal food processing. By connecting two positive/negative multiplier blocks in series, the proposed CWVM amplifies the input voltage twice. Owing to the series-connected bipolar topology, the proposed CWVM can achieve high conversion ratio with a small number of stages. In other words, the proposed CWVM can generate a high output voltage with small number of circuit components. To clarify circuit characteristics, simulation program with integrated circuit emphasis (SPICE) simulations and theoretical analysis are performed concerning the proposed CWVM.

\section{Circuit Configuration}

Fig.1 illustrates the block diagram of the CWVMs. Unlike conventional CWVMs, the proposed CWVM consists of a full-waveform rectifier and three voltage multiplier blocks (VMBs). Fig.2 shows an example of the circuit configuration of the proposed CWVM. As Figs. 1 and 2 show, the proposed CWVM amplifies the input voltage twice.

The operation principle of the proposed CWVM is as follows: First, the AC input $V_{A C}$ is full-wave rectified by the full-wave rectifier (FWR). Next, the non-overlapped twophase rectangular pulses $\Phi_{1}$ and $\Phi_{2}$ are provided by the clock pulse generator (CPG), where the full-wave rectified voltage $V_{c c}$ is used to generate $\Phi_{1}$ and $\Phi_{2}$. Then, the voltage $V_{c c}$ 
doubles by the first VMB, where the output voltages of the 1 st VMB are denoted by $V_{o 1}$ and $V_{o 2}$. Finally, by amplifying the voltage $V_{c c}$ again in the 2nd VMBs, the proposed CWVM generates a high stepped-up voltage about $3.5 \mathrm{kV}$. To reduce the ripple output voltage, the 2nd VMBs are connected in parallel.

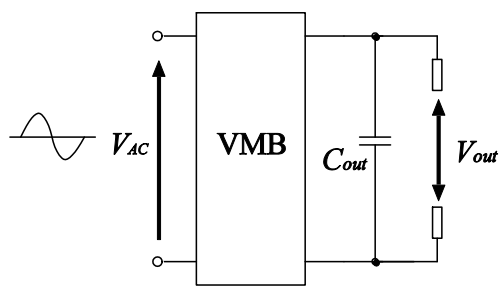

VMB: Voltage Multiplier Block

(a) Conventional CWVM [5], [8]

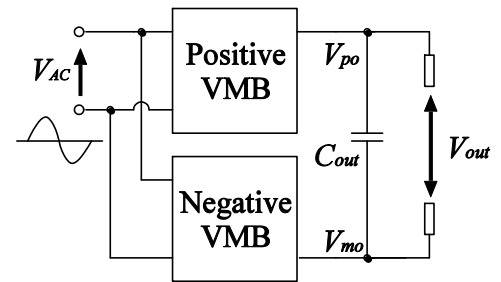

VMB: Voltage Multiplier Block

(b) Conventional CWVM [7]

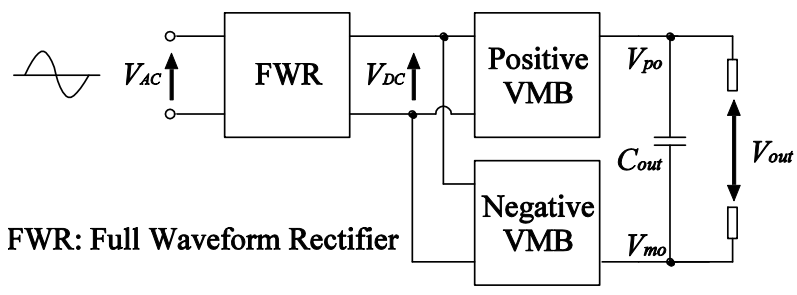

(c) Conventional CWVM [9]

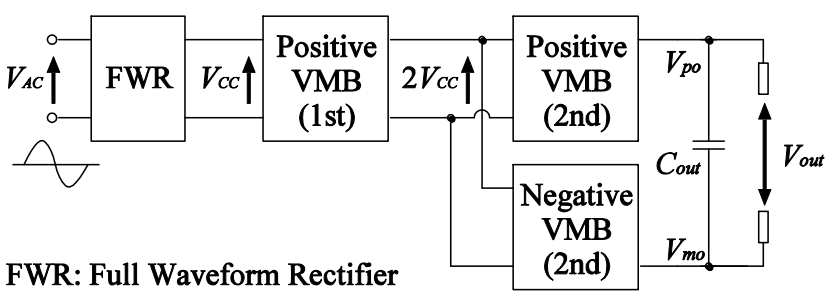

(d) Proposed CWVM

Figure 1. Block diagram of the CWVMs

Table 1 shows the comparison of output voltages between the proposed CWVM and conventional CWVMs. In Table 1, $\mathrm{N}(=1,2, \ldots)$ is the number of stages of the VMB and Vth denotes the threshold voltage of the diode switch. As Table 1 shows, the step-up gain of the proposed CWVM is higher than that of the conventional CWVMs. Concretely, the gain of the proposed CWVM is about double of that of the conventional multipliers reported in [7] and [9]. In other words, the number of stages of the proposed CWVM is about a half of that of the conventional CWVMs if the step-up gain is same. Therefore, the proposed CWVM can reduce the number of circuit components and circuit volume.

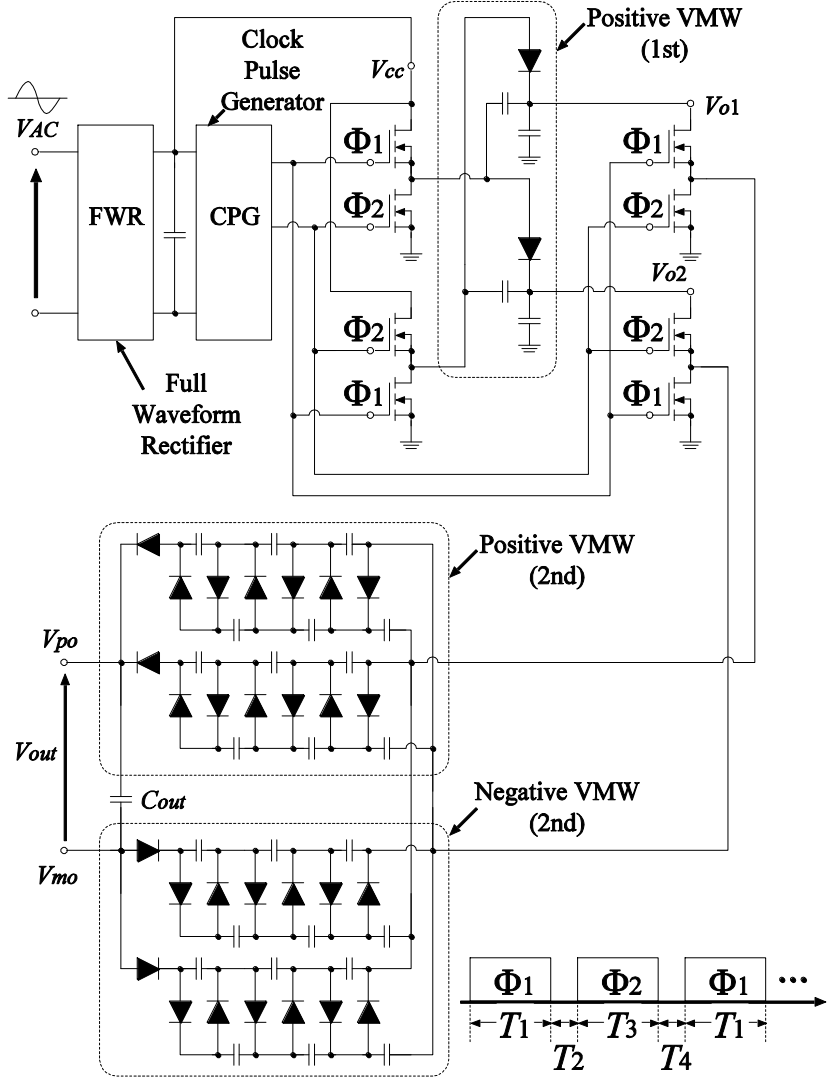

Figure 2. Circuit configuration of the proposed CWVM

TABLE I. COMPARISON OF OUTPUT VOLTAGES

\begin{tabular}{|c|c|}
\hline & Output Voltage \\
\hline $\begin{array}{c}\text { Proposed CWVM } \\
(N=3)\end{array}$ & $\begin{array}{r}V_{\text {out }}=2(2 N+1)\left(V_{c c}-V_{t h}\right) \\
+2(2 N)\left(V_{c c}-V_{t h}\right)-V_{t h}\end{array}$ \\
\hline $\begin{array}{c}\text { Conventional } \\
\text { CWVM [5], [8] }\end{array}$ & $V_{\text {out }}=2 N\left(V_{c c}-V_{t h}\right)$ \\
\hline $\begin{array}{c}\text { Conventional } \\
\text { CWVM [7], [9] }\end{array}$ & $\begin{array}{c}V_{\text {out }}=(2 N+1) V_{c c}-(2 N+1) V_{\text {th }} \\
+(2 N) V_{c c}-(2 N+1) V_{t h}\end{array}$ \\
\hline
\end{tabular}

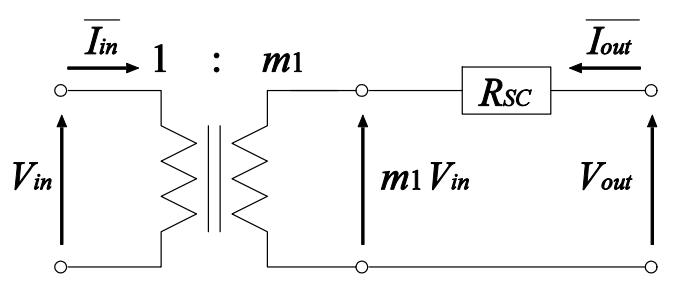

Figure 3. Four-terminal equivalent circuit

\section{Theoretical Analysis}

To analyze the characteristics of the proposed CWVM, theoretical analysis is performed, where the equivalent circuit is assumed as a four-terminal equivalent circuit reported in 


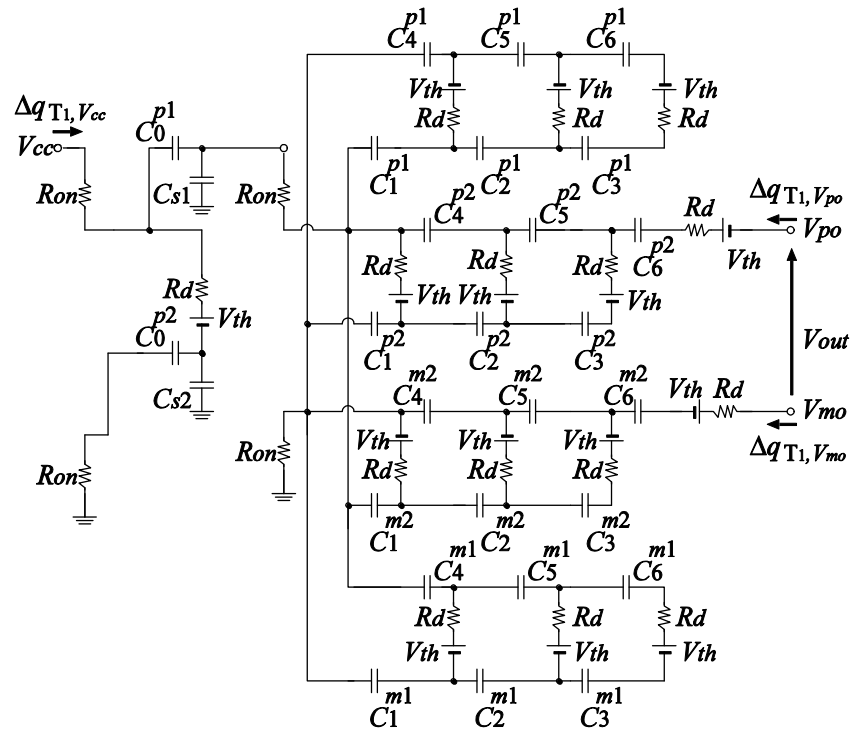

(a) State- $T_{1}$

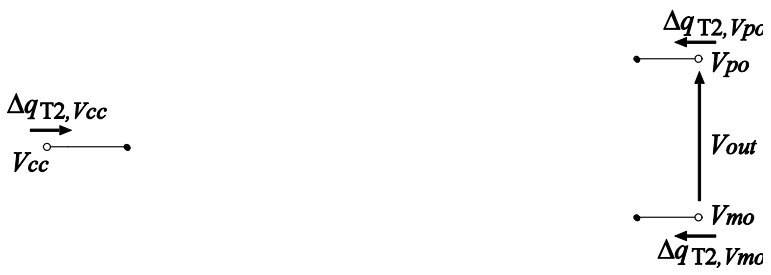

(b) State- $T_{2}$

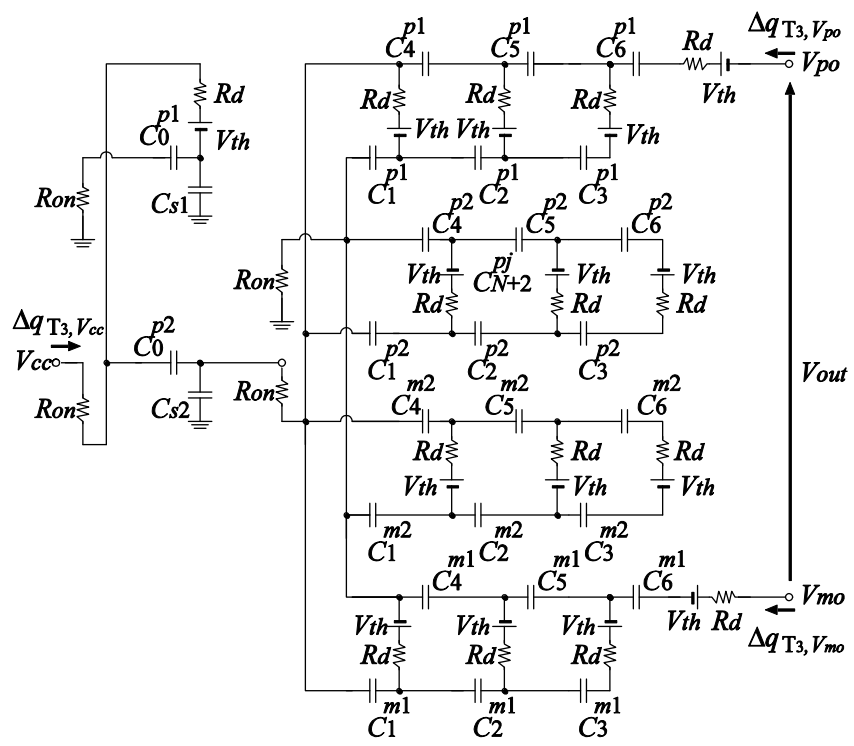

(c) State- $T_{3}$

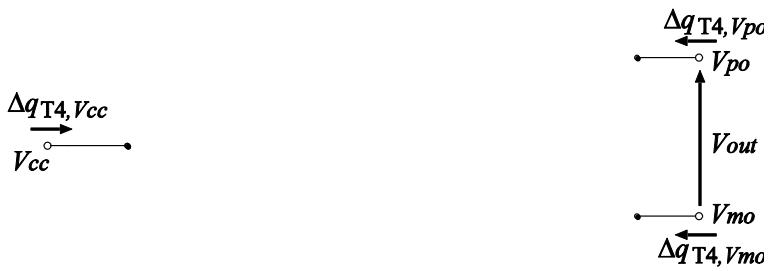

(d) State- $T_{4}$

Figure 4. Instantaneous equivalent circuits
[10]. Fig.3 shows the four-terminal equivalent circuit, where $m_{1}$ denotes the ratio of an ideal transformer and $R_{S C}$ is called the SC (switched capacitor) resistance.

In steady state, the instantaneous equivalent circuits of the proposed CWVM can be expressed by Fig.4, where $R_{\text {on }}$ denotes the on-resistance of the transistor switch, $R_{d}$ denotes the on-resistance of the diode, and $V_{t h}$ is the threshold voltage of the diode. In Fig.4, the differential value of the electric charge in $C_{k}^{p j}$ and $C_{k}^{m j}((k=0,1, \ldots, 6)$ and $(j=1,2))$ satisfies

$$
\sum_{i=1}^{4} \Delta q_{k, T_{i}}^{p j}=0 \quad \text { and } \quad \sum_{i=1}^{4} \Delta q_{k, T_{i}}^{m j}=0,
$$

where $\Delta q_{k, T i}{ }^{m j}$ and $\Delta q_{k, T i}^{p j}$ denote the electric charges of the $k$-th capacitor in State- $T_{i}$. The interval of State- $T_{i}$ satisfies the following conditions:

$$
T=\sum_{i=1}^{4} T_{i}, T_{1}=T_{3}=T_{s}, \text { and } T_{2}=T_{4}=\delta T,
$$

where $T$ is the period of clock pulses, $T_{i}(i=1, \ldots, 4)$ is the pulse width of $\Phi_{i}$, and $\delta$ is the parameter to determine the interval of State- $T_{2}$ and State- $T_{4}$. In State- $T_{1}$, the differential values of electric charges in the input and output terminals, $\Delta q_{T 1, V c c}, \Delta q_{T 1, V p o}$, and $\Delta q_{T 1, V m o}$, are expressed as

$$
\begin{aligned}
& \Delta q_{T_{1}, V_{c c}}=-\Delta q_{0, T_{1}}^{p 1}+\Delta q_{0, T_{1}}^{p 2}, \\
& \Delta q_{T_{1}, V_{p o}}=\Delta q_{6, T_{1}}^{p 2}, \text { and } \Delta q_{T_{1}, V_{m o}}=-\Delta q_{6, T_{1}}^{m 2} .
\end{aligned}
$$

In (3), the smoothing capacitors $C_{s 1}$ and $C_{s 2}$ are omitted to simplify the theoretical analysis.

On the other hand, in State- $T_{2}$, the differential values of electric charges in the input and output terminals, $\Delta q_{T 2, V c c}$, $\Delta q_{T 2, V p o}$, and $\Delta q_{T 2, V m o}$, are expressed as

$$
\Delta q_{T_{2}, V_{c c}}=\Delta q_{T_{2}, V_{p o}}=\Delta q_{T_{2}, V_{m o}}=0 .
$$

In the same way, the differential values of electric charges in State- $T_{3}$ and State- $T_{4}$ are expressed as

$$
\begin{gathered}
\Delta q_{T_{3}, V_{c c}}=-\Delta q_{0, T_{3}}^{p 2}+\Delta q_{0, T_{3}}^{p 1}, \\
\Delta q_{T_{3}, V_{p o}}=\Delta q_{6, T_{3}}^{p 1} \text {, and } \Delta q_{T_{3}, V_{m o}}=-\Delta q_{6, T_{3}}^{m 1}, \\
\text { and } \Delta q_{T_{4}, V_{c c}}=\Delta q_{T_{4}, V_{p o}}=\Delta q_{T_{4}, V_{m o}}=0 .
\end{gathered}
$$

Furthermore, using the Kirchhoff's current laws, the following equations are satisfied in the positive converter blocks:

$$
\begin{gathered}
\Delta q_{6, T_{1}}^{p j}=\Delta q_{5, T_{1}}^{p j}+\Delta q_{3, T_{1}}^{p j}, \\
\Delta q_{5, T_{1}}^{p j}=\Delta q_{4, T_{1}}^{p j}+\Delta q_{2, T_{1}}^{p j}-\Delta q_{3, T_{1}}^{p j}, \\
\Delta q_{6, T_{3}}^{p j}=-\Delta q_{3, T_{3}}^{p j}=\Delta q_{5, T_{3}}^{p j}+\Delta q_{2, T_{3}}^{p j}-\Delta q_{3, T_{3}}^{p j}, \\
\text { and } \Delta q_{5, T_{3}}^{p j}=\Delta q_{4, T_{3}}^{p j}+\Delta q_{1, T_{3}}^{p j}-\Delta q_{2, T_{3}}^{p j} .
\end{gathered}
$$

Therefore, we have

$$
\begin{aligned}
& \Delta q_{1, T_{1}}^{p j}=\left(\frac{3}{2}\right) \Delta q_{1, T_{1}}^{p j}=3 \Delta q_{3, T_{1}}^{p j} \\
& =-\Delta q_{4, T_{1}}^{p j}=-\left(\frac{3}{2}\right) \Delta q_{5, T_{1}}^{p j}=-3 \Delta q_{6, T_{1}}^{p j} .
\end{aligned}
$$

On the other hand, the following equations are satisfied in the negative converter blocks: 
Proc. of The Third Intl. Conf. On Advances in Applied Science and Environmental Technology - ASET 2015 Copyright $\odot$ Institute of Research Engineers and Doctors, USA .All rights reserved.

ISBN: 978-1-63248-084-2 doi: 10.15224/ 978-1-63248-084-2-71

$$
\begin{gathered}
\Delta q_{6, T_{1}}^{m j}=\Delta q_{5, T_{1}}^{m j}+\Delta q_{3, T_{1}}^{m j}, \\
\Delta q_{5, T_{1}}^{m j}=\Delta q_{4, T_{1}}^{m j}+\Delta q_{2, T_{1}}^{m j}-\Delta q_{3, T_{1}}^{m j}, \\
\Delta q_{6, T_{3}}^{m j}=-\Delta q_{3, T_{3}}^{m j}=\Delta q_{5, T_{3}}^{m j}+\Delta q_{2, T_{3}}^{m j}-\Delta q_{3, T_{3}}^{m j}, \\
\text { and } \Delta q_{5, T_{3}}^{m j}=\Delta q_{4, T_{3}}^{m j}+\Delta q_{1, T_{3}}^{m j}-\Delta q_{2, T_{3}}^{m j} .
\end{gathered}
$$

Therefore, we have

$$
\begin{aligned}
& \Delta q_{1, T_{1}}^{m j}=\left(\frac{3}{2}\right) \Delta q_{1, T_{1}}^{m j}=3 \Delta q_{3, T_{1}}^{m j} \\
& =-\Delta q_{4, T_{1}}^{m j}=-\left(\frac{3}{2}\right) \Delta q_{5, T_{1}}^{m j}=-3 \Delta q_{6, T_{1}}^{m j} .
\end{aligned}
$$

Using (3) - (6), the average input current and the average output currents can be obtained as

$$
\begin{gathered}
I_{c c}=\frac{\Delta q_{V_{c c}}}{T}=\frac{1}{T} \sum_{i=1}^{4} \Delta q_{T_{i}, V_{c c}}, \\
I_{p o}=\frac{\Delta q_{V_{p o}}}{T}=\frac{1}{T} \sum_{i=1}^{4} \Delta q_{T_{i}, V_{p o}}, \\
I_{m o}=\frac{\Delta q_{V_{m o}}}{T}=\frac{1}{T} \sum_{i=1}^{4} \Delta q_{T_{i}, V_{m o}}, \\
\text { and } \Delta q_{V_{o}}=\Delta q_{V_{p o}}=-\Delta q_{V_{m o}} .
\end{gathered}
$$

In (11), $\Delta q_{V c c}, \Delta q_{V p o}$, and $\Delta q_{V m o}$ are electric charges in $V_{c c}, V_{p o}$, and $V_{m o}$, respectively. Substituting (3) - (10) into (11), we have the relation between the input current and the output currents as follows:

$$
\begin{gathered}
I_{c c}=-14 I_{p o}+12 I_{m o}, \\
\text { where } \\
\Delta q_{V_{c c}}=-14 \Delta q_{V_{p o}}+12 \Delta q_{V_{m o}} \\
=-26 \Delta q_{V_{o}} .
\end{gathered}
$$

Next, let us consider the consumed energy $W_{T}$ in one period. Using (1) - (10), the consumed energy $W_{T}$ can be expressed as

$$
W_{T}=\sum_{i=1}^{4} W_{T_{i}}=2 W_{T_{1}}
$$$$
\text { where } \quad W_{T_{1}}=\frac{521\left(\Delta q_{V_{p o}}\right)^{2}}{2(1-2 \delta) T} R_{d}+\frac{1183\left(\Delta q_{V_{p o}}\right)^{k}}{2(1-2 \delta) T} R_{o n} .
$$

In (13), $W_{T i}$ denotes the consumed energy in State- $T_{i}$. Here, the consumed energy $W_{T}$ of the four-terminal equivalent circuit is defined as

$$
W_{T}:=\left(\frac{\Delta q_{V_{\text {out }}}}{T}\right)^{2} \cdot R_{S C} \cdot T .
$$

Therefore, from (13) and (14), we have the SC resistance as follows:

$$
R_{S C}=\left(\frac{521}{1-2 \delta}\right) R_{d}+\left(\frac{1183}{1-2 \delta}\right) R_{o n} .
$$

By combining (12) and (15), the equivalent circuit of the proposed CWVM can be obtained as Fig.5. The equivalent circuit of Fig. 5 is expressed by the following K-matrix:

$$
\left[\begin{array}{c}
V_{c c}-\frac{27}{26} V_{\text {th }} \\
I_{c c}
\end{array}\right]=\left[\begin{array}{cc}
\frac{1}{26} & 0 \\
0 & 26
\end{array}\right]\left[\begin{array}{cc}
1 & R_{S C} \\
0 & 1
\end{array}\right]\left[\begin{array}{c}
V_{\text {out }} \\
-I_{\text {out }}
\end{array}\right] .
$$

From (16), the output voltage and power efficiency of the proposed CWVM are obtained as

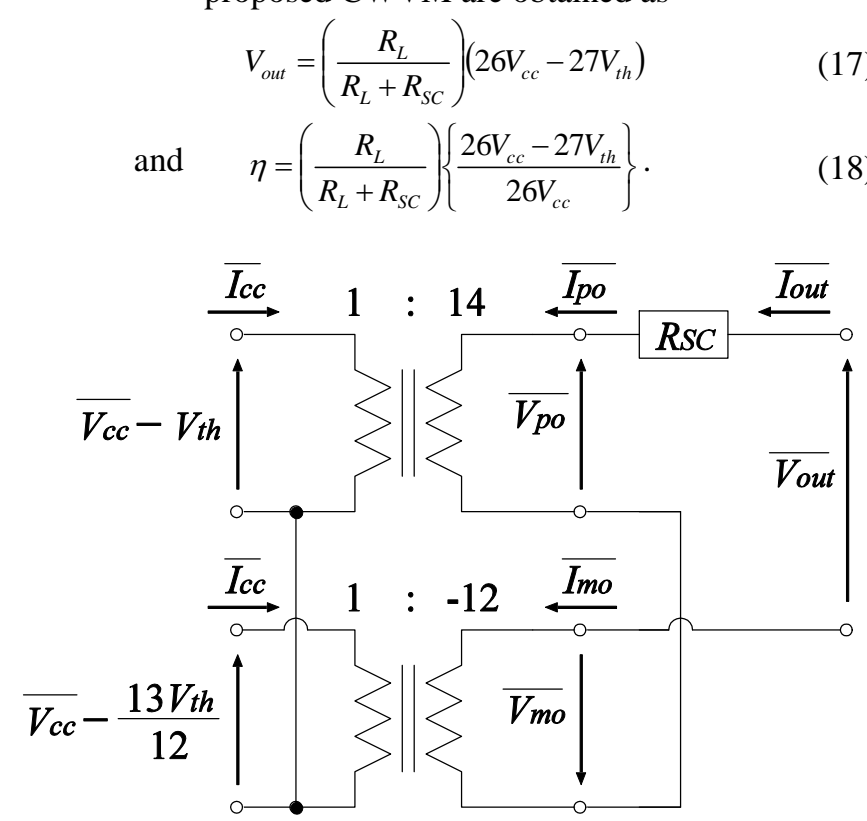

Figure 5. Equivalent circuit of the proposed CWVM

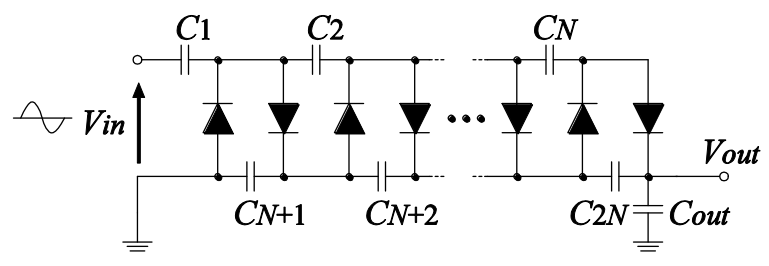

Figure 6. Traditional CWVM

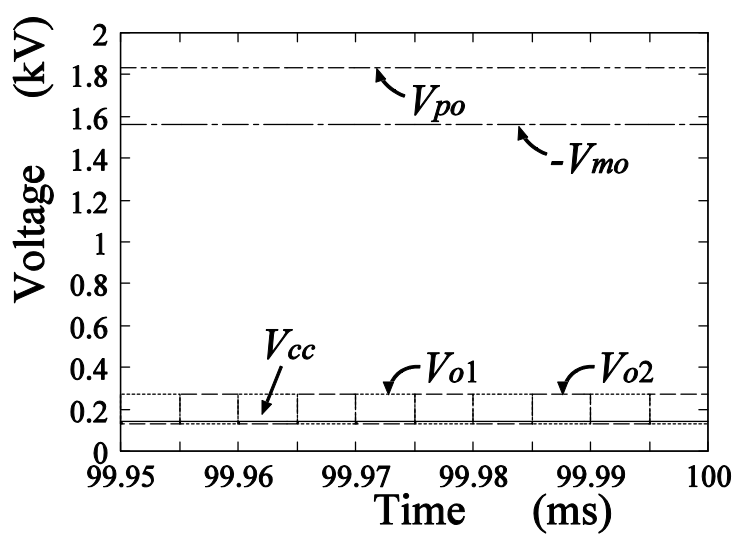

Figure 7. Simulated voltage of VMBs

\section{Simulation}

To clarify circuit characteristics, SPICE simulations are performed concerning the proposed CWVM of Fig. 2 and the traditional CWVM shown in Fig.6. Fig.7 shows the simulated output voltages of the VMBs in the proposed CWVM. In 
Fig.7, the SPICE simulation was performed under conditions that $N=3, V_{A C}=100 \mathrm{~V} @ 60 \mathrm{~Hz}, T=10 \mu \mathrm{s}, T_{s}=4.5 \mu \mathrm{s}, \delta=0.05$, $C_{k}^{p 1}=C_{k}^{p 2}=C_{k}^{m 1}=C_{k}^{m 2}=10 \mu \mathrm{F}$, and $C_{\text {out }}=100 \mu \mathrm{F}$. As Fig.7 shows, the input voltage $V_{c c}$ is converted twice by the VMBs (see Fig.2).

Fig.8 shows the simulated output $V_{\text {out }}$ of the CWVM. In Fig.8 (a), the output voltage of the proposed CWVM is $3.39 \mathrm{kV}$. The settling time of the proposed CWVM is less than $80 \mathrm{~ms}$. On the other hand, the output voltage of the traditional CWVM is $3.38 \mathrm{kV}$, where the parameter $N$ was set to 12 . The settling time of the traditional CWVM is less than 90s. Obviously, the settling time of the proposed CWVM is much shorter than that of the traditional CWVM.

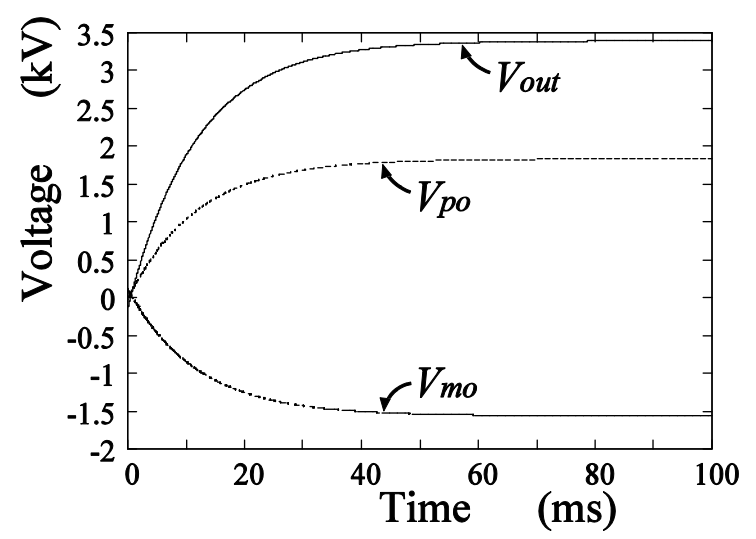

(a) Proposed CWVM

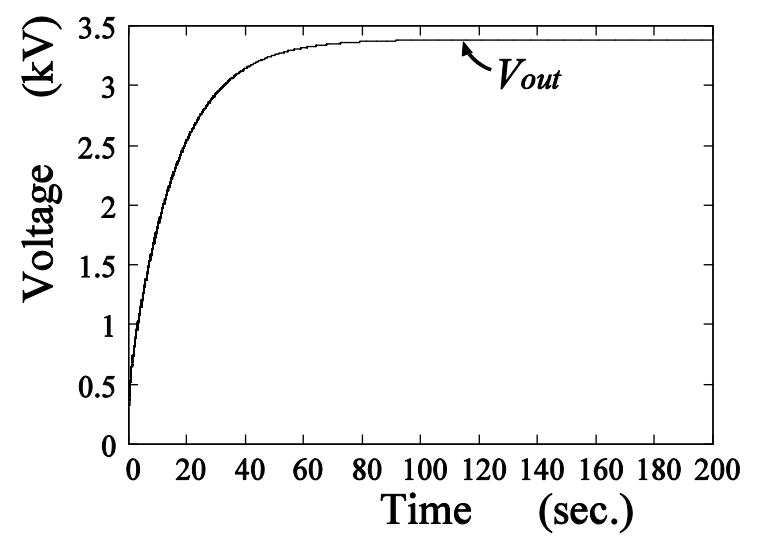

(b) Traditional CWVM

Figure 8. Simulated output voltage

\section{Conclusion}

For non-thermal food processing, a novel CWVM with series-connected bipolar topology has been proposed in this paper. Owing to the series-connected bipolar topology, the proposed CWVM can achieve higher step-up gain than conventional CWVMs.

The validity of circuit design was confirmed by SPICE simulations. By converting the input voltage $100 \mathrm{~V} @ 60 \mathrm{~Hz}$, about $3.5 \mathrm{kV}$ was generated by the proposed CWVM. The proposed CWVM can offer about $3.5 \mathrm{kV}$ output with a smaller number of stages than the traditional CWVM. Furthermore, the proposed CWVM can achieve high response speed. The settling time of the proposed CWVM is less than $80 \mathrm{~ms}$.

\section{Acknowledgment}

This research is supported by a grant from Urakami Foundation for Food and Food Culture Promotion.

\section{References}

[1] C. H. Zhang, T. Namihira, T. Kiyan, K. Nakashima, S. Katsuki, H. Akiyama, H. Ito, and Y. Imaizumi, "Investigation of shockwave produced by large volume pulsed discharge under water," IEEE Pulsed Power Conf., pp. 1377-1380, June 2005.

[2] Y. Miyafuji, K. Shimojima, S. Tanaka, K. Naha, T. Aka, H. Maehara, and S. Itoh, "Development of the pressure vessel for manufacturing the rice-powder using the underwater shock wave," Proc. of the ASME 2011 Pressure Vessels and Piping Conf., pp. 53-56, July 2011.

[3] K. Naha, K. Shimojima, Y. Miyafuji, and S.Itoh, "Design and development of pressure vessel for improvement of manufacturing ricepowder efficiency using underwater shock wave," Proc. of the ASME 2012 Pressure Vessels and Piping Conf., pp. 15-19, July 2012.

[4] S. Shinzato, Y. Higa, T. Tamaki, H. Iyama, and S. Itoh, "Computational simulation of underwater shock wave propagation using smoothed particle hydrodynamics," Materials Science Forum, vol. 767, pp. 86-91, July 2013.

[5] A. Lamantia, P. Maranesi, and L. Radrizzani, "The dynamics of the Cockcroft-Walton voltage multiplier," Proc. of the IEEE Power Electronics Specialists Conf., pp. 485-490, June 1990.

[6] J. Wang and P. Luerkens, "Complete model of parasitic capacitances in a cascade voltage multiplier in the high voltage generator," Proc. of the IEEE ECCE Asia Downunder, pp. 18-24, June 2013.

[7] S. Iqbal and R. Besar, "A bipolar Cockcroft-Walton voltage multiplier for gas lasers," American Journal of Applied Sciences, vol. 4, no.10, pp. 795-801, Jan. 2007.

[8] S. Iqbal, "A hybrid symmetrical voltage multiplier," IEEE Trans. Power Electronics, vol. 29, no.1, pp. 6-12, Jan. 2014.

[9] K. Eguchi, S. Pongswatd, S. Terada, and I. Oota, "Parallel-connected high voltage multiplier with symmetrical structure," Applied Mechanics and Materials, vol. 619, pp. 173-177, Aug. 2014.

[10] K. Eguchi, K. Fujimoto, and H. Sasaki, "A hybrid input charge-pump using micropower thermoelectric generators," IEEJ Trans. Electrical and Electronic Engineering, vol.7, no.4, pp. 415-422, July 2012. 